\title{
TREINAMENTO BIM NA QUATRE
}

\author{
Sandra Albino Ribeiro ${ }^{(1)}$, Rani De Moraes Soares ${ }^{(1)}$, Josyanne Pinto Giesta ${ }^{(2)}$ \\ (1) Quatre ensino especializado, Natal \\ (2) IFRN/ Instituto Federal de Educação, Ciência e Tecnologia do Rio Grande do Norte, Natal
}

\begin{abstract}
Resumo
O Building Information Modeling (BIM), visto como uma metodologia promissora na indústria da construção civil, possui um aporte teórico amplo e complexo com conceitos, ferramentas, fluxos de trabalho e procedimentos, os quais devem ser compreendidos e aplicados pelos agentes desse setor. Ao observar a literatura percebe-se que muito já tem sido discutido e publicado sobre a adoção do BIM em cursos de graduação, mas quando se compara a outros modos de aprendizagem as pesquisas são incipientes. Considerando essa lacuna, o presente trabalho tem por objetivo descrever e analisar uma escola de treinamentos, que oferta cursos de aperfeiçoamento profissional em BIM as áreas de Arquitetura, Engenharia e Construção, na cidade de Natal, estado do Rio Grande do Norte, Brasil, tendo como público, graduandos e graduados nessas áreas. O método utilizado foi estudo de caso e os resultados da pesquisa, mostram que a aprendizagem BIM desses treinamentos tem maior enfoque no campo da tecnologia. Porém, se observa um direcionamento na abordagem de competências que possibilitem a compreensão de processos e estratégias para implementação do BIM em escritórios e construtoras. As principais contribuições deste estudo são o fomento reflexões que conduzam a criação e melhoria de treinamentos de cursos livres para aperfeiçoamento profissional em BIM.
\end{abstract}

\section{Introdução}

No Brasil, a exemplo de outros países, a adoção do BIM é cada vez mais reconhecida como o melhor caminho para inovação da indústria da construção civil, haja vista que essa vertente possibilita novos métodos de trabalhos e tecnologias computacionais as quais geram ganhos em todo ciclo de vida da edificação: da concepção, passando pelo planejamento e gerenciamento da obra, operação (na gestão e na manutenção) e reuso, retrofit ou demolição.

Diante da sua abrangência e complexidade, é possível encontrar diferentes conceitos sobre essa filosofia de trabalho. Alguns autores a definem a partir da combinação de um conjunto de tecnologias, processos e políticas que alteram resultados e papéis da construção civil [1]. Outros ressaltam os seus aspectos como a colaboração e a integração que ocorrem por meio de modelos 
digitais precisos, por sua vez baseados em objetos paramétricos [2]. Apesar de existir variações, a literatura é unânime ao reconhecer que se trata de uma nova metodologia de trabalho com muito mais vantagens, comparada aos processos tradicionais.

Em meio a isso, há um encadeamento natural de adequação dos profissionais aos processos de trabalho em BIM, por consequência, emerge a necessidade por capacitações. Sendo essa demanda reforçada nos últimos anos pela publicação do Decreto $\mathrm{N}^{\circ} 9^{\circ} 377$, de 17 de maio de 2018 [3], que foi revogado pelo Decreto $N^{\circ} 9.983$ de 22 de agosto de 2019 [4]. Ambos estabelecem a Estratégia Nacional de Disseminação do Building Information Modeling através de 9 objetivos específicos, entre eles o número: "IV - Estimular a capacitação em BIM". Ressalta-se que em 5 de junho de 2017 foi lançado o primeiro Decreto [5] que instituiu o Comitê Estratégico de Implementação do Building Information Modelling e deu origem aos Decretos de 2018 e 2019. Recentemente, foi publicado o Decreto $\mathrm{N}^{\circ}$ 10.306, de 02 de abril de 2020 [6], que estabelece a utilização do BIM na execução direta e indireta de obras e serviços de engenharia em órgãos na esfera pública federal.

As instituições acadêmicas, tanto em nível nacional quanto internacional, já estão discutindo na literatura o ensino do BIM, as funções e as responsabilidades dos discentes, assim como as competências, especialmente dentro dos cursos de arquitetura e engenharia [7,8,9]. Nos últimos anos, ocorreram dois eventos I e II Encontro Nacional de Ensino de BIM (ENEBIM), respectivamente nas cidades de Campinas (2018) e de Fortaleza (2019), que reuniram a comunidade científica, docentes e especialistas em torno de discussões sobre o ensino e aprendizagem dessa vertente. Nos dois encontros, ficou claro que para vencer os desafios que a implementação do BIM apresenta é importante capacitar a mão de obra, apesar disso grande parte das publicações neste e em outros eventos tem enfoque quase exclusivo sobre o ensino de BIM na graduação, ou, com algumas exceções, pós-graduação, sendo raros os casos de estudos sobre escolas de aperfeiçoamento profissional e cursos livres na área.

Nesse sentido, o presente trabalho objetiva descrever e analisar uma escola de cursos livres, que oferta capacitações em BIM para profissionais e futuros profissionais na área de Arquitetura, Engenharia e Construção (AEC) na cidade de Natal/RN.

\section{Método}

Este trabalho pode ser classificado como uma pesquisa descritiva e os procedimentos metodológicos adotados foram a pesquisa bibliográfica, a pesquisa documental e o estudo de caso. Na pesquisa bibliográfica foram elaboradas revisões teóricas sobre o ensino de BIM. A pesquisa documental se baseou nos planos de ensino, ementas das disciplinas e atividades que revelassem o espectro do conteúdo BIM trabalhado em sala de aula. Para o estudo de caso foram escolhidos os treinamentos "A" e "B" da escola Quatre Ensino Especializado situada em Natal/RN. Essa escola foi selecionada por ser "uma das instituições mais antigas a ofertar cursos em BIM na região Nordeste" e esses treinamentos por apresentarem maior carga horária de assuntos relacionados ao BIM. Durante o estudo de caso, a coleta de dados ocorreu por meio de entrevista semi-estruturada com todos os coordenadores e com três instrutores que estão mais tempo na escola e ministram a maior parte do conteúdo dos treinamentos escolhidos, além de serem realizadas observações não participantes a fim de averiguar a dinâmica em sala de aula. Após esses procedimentos, todas as informações obtidas foram sistematizadas, relacionadas e analisadas visando alcançar o objetivo da pesquisa. 


\section{Os caminhos do ensino de BIM}

Várias pesquisas brasileiras buscam compreender o impacto da incorporação do BIM no ensino de Arquitetura e Engenharia Civil ou sugerem caminhos para esta transformação [10]. $\mathrm{O}$ presente trabalho buscou publicações que se encaixem na segunda proposição e que enumerem competências (conteúdos, habilidades e atitudes) necessárias para atuação do profissional em processos BIM. Além de exibirem instrumentos que averiguem o nível de proficiência em BIM e possibilitem adaptações a um modo de aprendizagem diferente da graduação.

Uma pesquisa que se encaixou nos quesitos da revisão bibliográfica é a Tese de Doutorado [7] da Maria Bernardete Barison, defendida em 2015 na Poli-USP. O referido trabalho mapeou e analisou mais de 100 instituições que ensinavam BIM nos cursos de Arquitetura, Engenharia Civil e Gerenciamento da Construção, sendo grande parte dessas escolas localizadas nos EUA. Com isso, descreveu três Níveis de Proficiência em BIM (NPBIM): Introdutório, Intermediário e Avançado. Cada um desses níveis apresenta métodos e recursos de ensino e aprendizagem próprios, de modo a desenvolver as competências para três novos perfis profissionais no cenário BIM: Modelador, Analista e Gerente. Para esses novos perfis foram elencados 33 principais funções e responsabilidades que se traduzem em especialidades BIM e estão distribuídas nas áreas de consultoria, indústria, ambiente virtual de aprendizagem e empresas (eixo profissional e gerencial).

No âmbito internacional, o Collaborative BIM Education Framework foi proposto pelo professor e pesquisador Bilal Succar [9], em conjunto com uma iniciativa nacional na Austrália, e abrange todos os modos de aprendizado. Essa estrutura permite sistematizar os domínios do BIM, possibilitando assim compreendê-lo, disseminá-lo e implementá-lo gradualmente. Além disso, pode ser visto como um elo da compreensão referente ao BIM, entre a academia e a indústria. O Collaborative BIM Education Framework contempla 6 componentes sendo eles respectivamente: 1) Identificar competências; 2) Classificar competências; 3) Criar Módulos de Aprendizagem BIM; 4) Framework Industrial; 5) Framework Acadêmico; 6) Criar um Instituto BIM.

Os 3 primeiros componentes tratam das Competências Individuais BIM [11] que representam a combinação de conhecimento, habilidade, experiência, em alguns casos atitudes e tratamentos pessoais como liderança. Elas estão agrupadas nas categorias: Gerencial; Funcional; Técnica; Suporte; Administração; Operação; Implementação; Pesquisa e desenvolvimento; e Essenciais. Acerca desse termo, tem-se um binário: competente ou incompetente. Mas, de acordo com Succar, entre esses extremos há nuances e níveis incrementais de especialização que afere a profundidade da compreensão conceitual e a extensão da experiência prática necessária para realizar uma atividade bem definida. São esses níveis divididos em cinco: 0 - Nenhum; 1 Básico; 2 - Intermediário; 3 - Avançado; 4 - Expert.

As pesquisas de Barison e de Succar indicam que o sucesso da implementação do BIM no mercado está relacionado a aprendizagem BIM e que o ensino de BIM deve ir além do ensino de softwares. Os autores citam também a importância de compreender os novos perfis profissionais, as competências e a proficiência no cenário BIM. Apesar de trazerem diferentes proposições, eles expressam que ensinar essa nova vertente é algo complexo e quando ela ocorrer deve ser feita de maneira gradual e contínua. Em meio a isso, o presente trabalho definiu que o enfoque seria a análise de componentes e traria a abordagem para a análise do que está sendo ensinado, ao invés de como, onde e quando a aprendizagem ocorreria. $\mathrm{Na}$ análise buscouse compreender o espectro BIM englobado e a partir disso listar as funções, responsabilidades, 
competências e o nível de proficiência. A descrição, a análise e os seus resultados são colocados a seguir.

\section{Estudo de caso}

A escola Quatre Ensino Especializado oferece cursos livres desde 2010, mas com o uso do BIM a partir de 2011, sendo pioneira nesse tipo de ensino no Nordeste. A modalidade cursos livres tem por base legal o Decreto Presidencial $N^{\circ} 5.154$ de 23 de julho de 2004 e tem por objetivo oferecer qualificação profissional, inclusive formação inicial e continuada. Os cursos podem ocorrer no nível médio, graduação e pós-graduação e estarem nas mais diversas áreas de atuação no mercado de trabalho. Apesar disso, a instituição de ensino estudada oferece cursos apenas para a área de Arquitetura, Engenharia e Construção Civil.

De acordo com a coordenação da escola, no primeiro ano (2011) foram certificados 45 alunos e no último ano (2019) foram certificados 305 alunos em cursos cuja tematica principal é o BIM. A coordenação informou que a procura por essa modalidade passou a ser intensificada a partir de 2015 e por essa razão a escola passou por várias adequações físicas e organizações para atender a essa nova demanda.

No período da pesquisa (2019), foi delimitado como objeto de estudo dois de seus cursos que serão denominados como treinamentos $\mathrm{A}$ e $\mathrm{B}$, sendo que $\mathrm{B}$ se enquadra na modalidade de treinamentos corporativos, ou seja, quando todos os alunos trabalham em uma mesma empresa. A escolha foi dada por esses cursos apresentarem a maior carga horária, por seus módulos (15h) terem relação direta com o BIM e por ser exigido o desenvolvimento de um projeto piloto para o recebimento do certificado de 300h (curso A com 10 módulos) e 345h (curso B com 13 módulos). Em ambos os cursos são desenvolvidos modelos BIM arquitetônicos, estruturais, de sistemas prediais e do terreno, sendo abordados os seguintes usos do modelo [12]: Documentação 2D; Detalhamento 3D; Representação construída; Topografia; Design autoral; Planejamento de construção; Detecção de interferência; Simulação de realidade aumentada; Simulação de realidade virtual; Análise de construtibilidade; Extração de quantitativos; Análise do terreno; Análise solar; e Análise estrutural. A Figura 1 apresenta uma das atividades desenvolvidas durantes os treinamentos.

As aulas ocorriam presencialmente, durante a semana ou nos fins de semana, e nelas são utilizadas as ferramentas Autodesk Revit $\AA$, Autodesk Robot ${ }^{\circledR}$, Autodesk Navisworks $®$, a plataforma A360®, BIMSight ${ }^{\circledR}$, Enscape ${ }^{\circledR}$ e o aplicado de Realidade Aumentada Augin ${ }^{\circledR}$. Cada turma contém no máximo 18 alunos com diferentes perfis. O curso B tem como diferencial 03 módulos específicos que apresentam conteúdos relacionados aos fundamentos e aos processos em BIM. Esses módulos ocorrem através da exposição teórica, do desenvolvimento de um Plano de Execução BIM, da criação de um template, da elaboração de vários protocolos os quais visam a orientação quanto ao processo de modelagem e a personalização do fluxo de trabalho. Antes de iniciar o treinamento corporativo são coletados dados que permitirão a compreensão dos processos e das tecnologias existentes na organização. Com isso é possível definir se o treinamento $\mathrm{B}$ seguirá ou não a ordem do treinamento $\mathrm{A}$ e quando serão inseridos os módulos específicos (B1, B2 e B3). Ver Figura 2. 

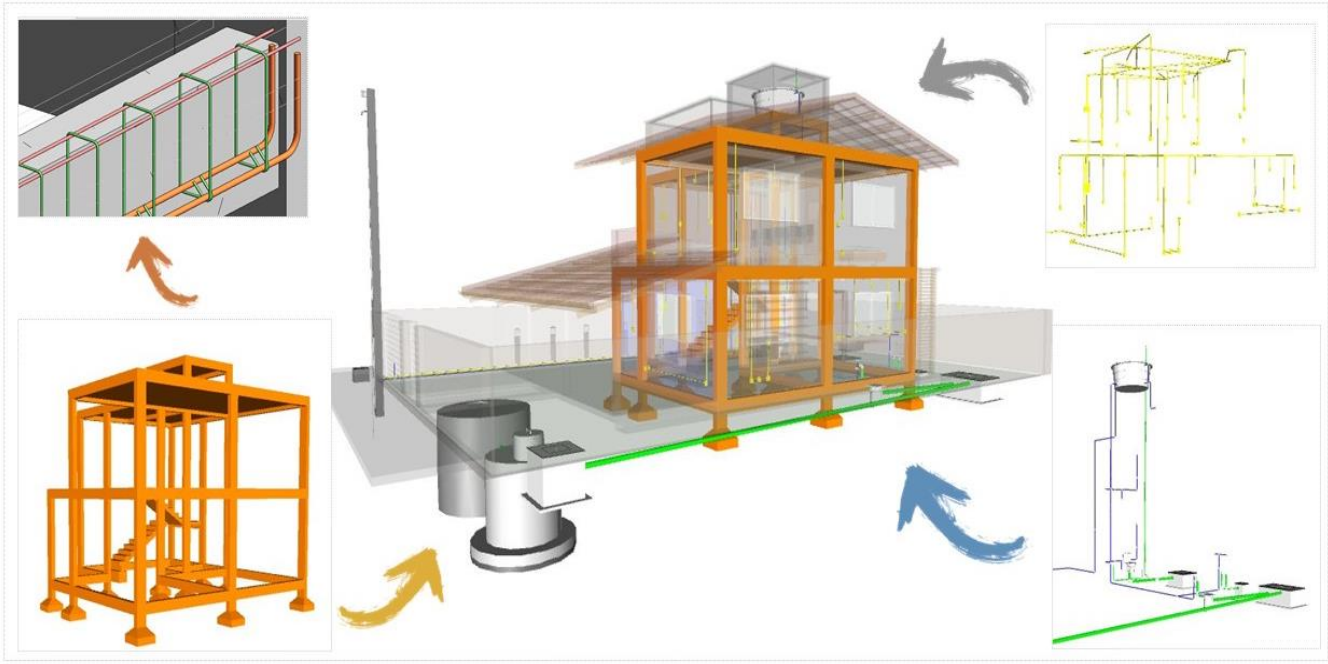

Figura 1: Modelos BIM desenvolvidos durante os treinamentos.

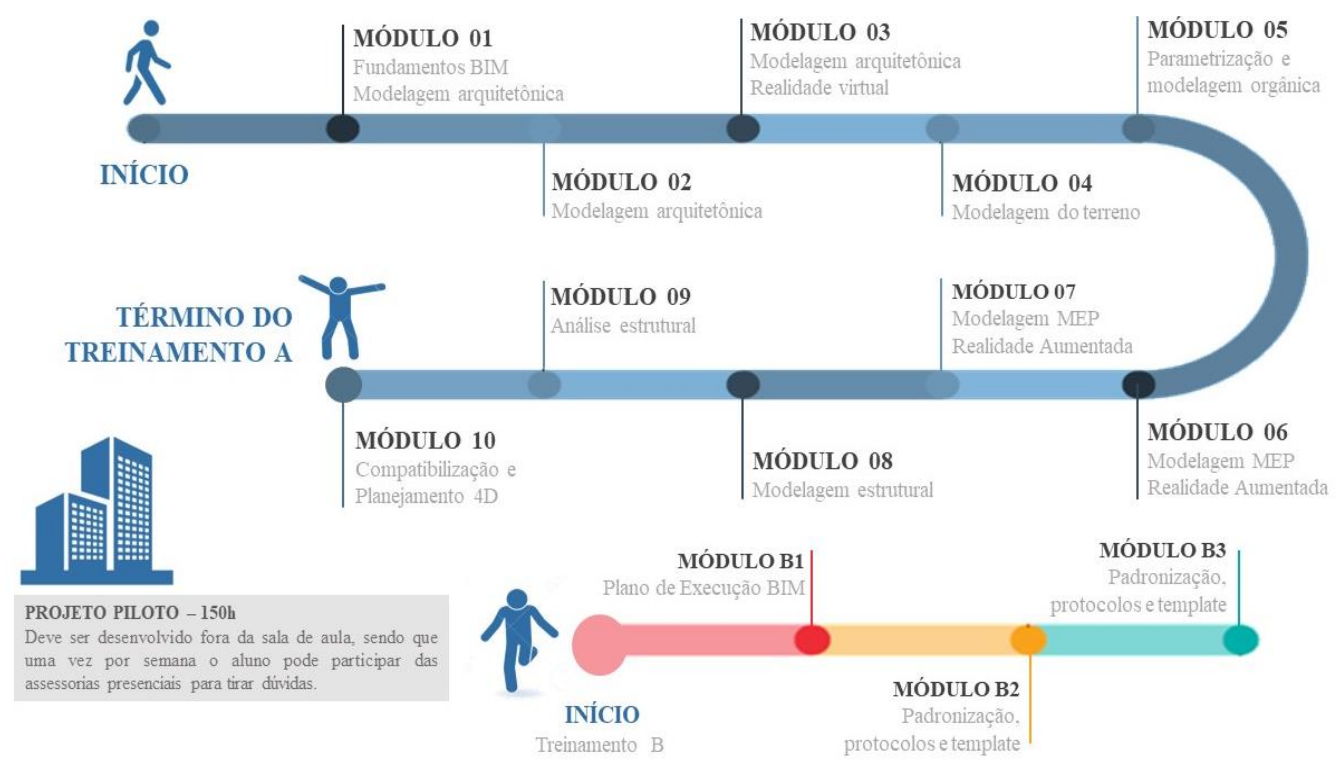

Figura 2: Fluxograma dos treinamentos A e B.

\section{Resultados e discussões}

Com base nos estudos de Barison [7], foram identificadas as funções e responsabilidades abordadas nos treinamentos analisados. Já sob a ótica de Succar $[9,11]$ foi possível detectar e classificar as Competências Individuais BIM existentes. As Tabelas 1 e 2 ilustram os resultados obtidos.

A partir desses dados, pode-se afirmar que a aprendizagem BIM do treinamento A tem como maior enfoque o campo da tecnologia, especialmente nas aplicações práticas (3D, 4D e 5D). No treinamento B, verificou-se um direcionamento para os campos de processos, haja visto que foi identificado o desenvolvimento de habilidades que promovem a compreensão e o desenho 
de processos, assim como o entendimento e a prática de estratégias para implementação de BIM em escritórios e construtoras.

Para os próximos anos a escola pretende inserir as ferramentas digitais Autodesk Dynamo®, Autodesk Civil 3D®, Autodesk Infraworks ${ }^{\circledR}$, Autodesk Formit ${ }^{\circledR}$ e Twinmotion ${ }^{\circledR}$. Após observar os resultados da pesquisa, a empresa se mostrou inclinada a adotar outras funções, responsabilidades e competências individuais do BIM. Além de sinalizar que irá elaborar um BIM Education Framework e focar no desenvolvimento de módulos de aprendizagem BIM, os quais poderão auxiliar todos os componentes analisados, tendo como enfoque outros campos além da tecnologia.

Tabela 1: Associação das funções e responsabilidades com os Treinamentos da Quatre. Adaptado de [7]

\begin{tabular}{|c|c|c|}
\hline Campo de atuação & $\begin{array}{l}\text { Especialista BIM } \\
\text { (Funções e responsabilidade) }\end{array}$ & $\begin{array}{l}\text { Treinamento e } \\
\text { Módulo }\end{array}$ \\
\hline Consultoria & $\begin{array}{l}\text { Consultor BIM operacional } \\
\text { (Executar o plano de implementação BIM) }\end{array}$ & $\begin{array}{c}\mathrm{B} \\
(\mathrm{B} 1, \mathrm{~B} 2, \mathrm{~B} 3)\end{array}$ \\
\hline \multirow{7}{*}{$\begin{array}{l}\text { Empresas: } \\
\text { eixo profissional }\end{array}$} & $\begin{array}{l}\text { Modelador BIM (Criar, desenvolver modelos } \\
\text { BIM e extrair documentação destes) }\end{array}$ & $\begin{array}{l}\text { A e B } \\
(01 \text { a } 09)\end{array}$ \\
\hline & $\begin{array}{l}\text { Modelador 3D (Criar geometrias nos modelos } \\
\text { e trabalhar com equipe de projeto) }\end{array}$ & $\begin{array}{c}\text { A e } B \\
(05 \text { e } 10)\end{array}$ \\
\hline & $\begin{array}{l}\text { Modelador de instalações (Desenvolver } \\
\text { projetos de instalações prediais detalhando } \\
\text { um modelo 3D) }\end{array}$ & $\begin{array}{c}\text { A e B } \\
(06 \text { e } 07)\end{array}$ \\
\hline & $\begin{array}{l}\text { Modelador de custos (Inserir no modelo - 5D } \\
\text { - informações sobre processos construtivos e } \\
\text { recursos) }\end{array}$ & $\begin{array}{c}\text { A e B } \\
(02,03 \text { e } 04)\end{array}$ \\
\hline & $\begin{array}{l}\text { Modelador de fabricação (Criar os modelos } \\
\text { 3D para a fabricação a partir do modelo de } \\
\text { construção) }\end{array}$ & $\begin{array}{l}\text { A e B } \\
(05)\end{array}$ \\
\hline & $\begin{array}{l}\text { Analista BIM (Fazer análises e simulações } \\
\text { baseadas no modelo) }\end{array}$ & $\begin{array}{c}\text { A e B } \\
(05,09 \text { e } 10)\end{array}$ \\
\hline & $\begin{array}{l}\text { Facilitador ou Piloto BIM (Auxiliar pessoas a } \\
\text { visualizarem o modelo BIM) }\end{array}$ & $\begin{array}{c}\text { A e B } \\
(01,04,05 \text { e } 10)\end{array}$ \\
\hline \multirow{2}{*}{$\begin{array}{l}\text { Empresas: } \\
\text { eixo gerencial }\end{array}$} & $\begin{array}{l}\text { Gerente BIM do escritório (Implementar } \\
\text { BIM no escritório e coordenar equipes de } \\
\text { projeto) }\end{array}$ & $\begin{array}{c}\mathrm{B} \\
(\mathrm{B} 1, \mathrm{~B} 2, \mathrm{~B} 3)\end{array}$ \\
\hline & $\begin{array}{l}\text { Coordenador da construtora (Estabelecer } \\
\text { padrões e processo BIM) }\end{array}$ & $\begin{array}{c}\mathrm{B} \\
(\mathrm{B} 1, \mathrm{~B} 2, \mathrm{~B} 3)\end{array}$ \\
\hline
\end{tabular}


Tabela 2: Associação das competências BIM com os Treinamentos da Quatre. Adaptado de [11]

Competências Individuais BIM

Conhecimento: Habilidades

Gerenciais: As habilidades de tomada de decisão que orientam a seleção / adoção de estratégias e iniciativas de longo prazo. Incluem Liderança, Planejamento Estratégico, Gestão Organizacional etc.
Treinamento e

Módulo

(B1, B2, B3)

Administrativas: As atividades organizacionais do dia-a-dia, conforme necessário para atender e manter objetivos estratégicos. Incluem Licitações e Aquisições, Administração de Contratos, RH e Não se aplica Recrutamento etc.

Funcionais: As habilidades não técnicas e gerais necessárias para iniciar, gerenciar e entregar projetos. As Competências Funcionais incluem Não se aplica Colaboração, Facilitação, Gerenciamento de Projetos etc.

Competências operacionais: Os esforços individuais diários e práticos necessários para entregar um projeto ou parte / aspecto de um projeto. A e B Competências Operacionais incluem Design, Análise, Simulação, (01 a 10)

Quantificação, Estimativa etc.

Competências técnicas: As habilidades individuais necessárias para gerar as entregas do projeto em disciplinas e especialidades. As A e B Competências técnicas incluem modelagem, desenho, gerenciamento de (01 a 10) modelos etc.

Competências de Implementação: As atividades necessárias para introduzir conceitos e ferramentas BIM em uma organização. Incluem Desenvolvimento de Componentes, Gerenciamento de Biblioteca BIM, (B1, B2, B3) Padronização etc.

Competências de Suporte: As Competências de Suporte são as habilidades necessárias para manter a tecnologia da informação e os A e B sistemas de comunicação. Incluem gerenciamento de arquivos e de rede, seleção e implantação de hardware, solução de problemas de software etc.

Competências de Pesquisa e Desenvolvimento: As habilidades necessárias para avaliar os processos existentes, investigar novas soluções e facilitar sua adoção - dentro da organização ou pela indústria maior. Não se aplica Incluem Facilitação da Mudança, Engenharia do Conhecimento, Ensino e Coaching etc.

Competências Essenciais: Especialidade de um indivíduo, experiência geral (em termos de meses / anos), exposição do mercado (em termos de geografia) e experiência em projetos (em termos de tipos de projetos,

Não se aplica tamanhos e orçamentos). 


\section{Conclusão}

Apesar desta pesquisa contribuir para o entendimento do ensino de BIM, especialmente, por descrever e analisar um modo de aprendizagem diferente da graduação ainda existem muitas lacunas. Sugere-se para futuros trabalhos uma investigação sobre as demandas espaciais e os recursos digitais e físicos utilizados nesse tipo de organização, além de realizar entrevistas com os formandos a fim de verificar o seu grau de satisfação com a formação obtida, se estão a utilizar profissionalmente o BIM e de que forma os cursos contribuíram para o seu desempenho profissional. Recomenda-se também examinar outras escolas de cursos livres, utilizar outros métodos como o de Checucci [8], apurar as dificuldades encontradas por alunos e professores e até mesmo participar da criação do BIM Education Framework da escola estudada.

Refletindo sob o contexto local e quiçá nacional, conclui-se que a maior demanda do mercado ainda é pelo uso das ferramentas BIM, mas nota-se que há uma inclinação por capacitações que possibilitem um melhor entendimento quanto a implementação e processos BIM. Vale destacar que a adoção do BIM é algo desafiador tanto para escolas quanto para construtoras e escritórios, pois implica em mudanças culturais significativas na área da construção civil e abrange além do campo tecnológico os campos de processos e políticas.

\section{Referências}

[1] SUCCAR, B.; KASSEM, M. Macro-BIM adoption: Conceptual structures. Automation in Construction, [s.1.], v. 57, p. 64- 79, 2015. ISSN: 0926-5805, DOI: https://doi.org/10.1016/j.autcon.2015.04.018

[2] C. M. Eastman, P. Teicholz, R. Sacks, and K. Liston, BIM handbook : a guide to building information modeling for owners, managers, designers, engineers, and contractors. Hoboken, N.J.: Wiley, 2008.

[3] JusBrasil. Decreto de 17 de maio de 2019. Disponível em: < http://www.planalto.gov.br/ccivil_03/_Ato2015-2018/2018/Decreto/D9377.htm> Acesso em: 02 fev. 2020.

[4] JusBrasil. Decreto de 22 de agosto de 2019. Disponível em: < http://www.planalto.gov.br/ccivil_03/_ato2019-2022/2019/decreto/D9983.htm> Acesso em: 14 jan. 2020.

[5] JusBrasil. Decreto de 5 de junho de 2017. Disponível em: < http://www.planalto.gov.br/ccivil_03/_Ato2015-2018/2017/Dsn/Dsn14473.htm> Acesso em: 02 fev. 2020.

[6] JusBrasil. Decreto de 2 de abril de 2020. Disponível em: < http://www.planalto.gov.br/ccivil_03/_ato2019-2022/2020/decreto/D10306.htm> Acesso em: 31 jul. 2020.

[7] BARISON, M. B., " Introdução de Modelagem da Informação da Construção - BIM - no currículo: uma contribuição para a formação do projetista " Tese de Doutoramento, Faculdade Politécnica, Universidade de São Paulo, São Paulo, Brasil, 2015. Disponível em: $<$ https://teses.usp.br/teses/disponiveis/3/3146/tde-21032016-101815/pt-br.php> Acesso em: 14 jan. 2020.

[8] CHECCUCCI, E. De S.., " Ensino-aprendizagem de BIM nos cursos de graduação em engenharia civil e o papel da expressão gráfica neste contexto " Tese de Doutoramento, 
Faculdade de Educação, Universidade Federal da Bahia, Salvador, Brasil, 2014. Disponível em: < http://repositorio.ufba.br/ri/handle/ri/15295 > Acesso em: 14 jan. 2020.

[9] B. Succar, W. Sher, A Competency knowledge-base for BIM learning, Australasian Journal of Construction Economics and Building - Conference Series, vol. 2, pp. 1-10, Abril 2014. doi: 10.5130/ajceb-cs.v2i2.3883

[10]RUSCHEL, R.; CUPERSCHMID, A. R. M. BIM como expressão atual da inovação no ensino. VEncontro da Associação Nacional de Pesquisa e Pós-Graduação em Arquitetura e Urbanismo - ANAIS, v. 8, 2018. ISSN: ISSN 2358-6214

[11] B. Succar, W. Sher, A. Williams, An integrated approach to BIM competency assessment, acquisition and application, Automation in Construction 35 (2013) 174-189, http://dx.doi.org/10.1016/j.autcon.2013.05.016.

[12] Succar, B., Saleeb, N., Sher, W. (2016), Model Uses: Foundations for a Page 2 of 12 Modular Requirements Clarification Language, Australasian Universities Building Education (AUBEA2016), Cairns, Australia, July 6-8, 2016. Disponível em: <https://www.researchgate.net/publication/303013287_Model_Uses_Foundations_for_a_ Modular_Requirements_Clarification_Language> Acesso em: 30 de jun de 2020. 